\title{
Development of a drug resistance model for hepatoblastoma
}

\author{
CARMEN EICHER, ALEXANDER DEWERTH，BETTINA KIRCHNER，STEVEN W. WARMANN, \\ JÖRG FUCHS and SORIN ARMEANU-EBINGER
}

Department of Pediatric Surgery, University Children's Hospital, Hoppe-Seyler-Strasse 3, 72076 Tuebingen, Germany

Received August 25,2010; Accepted October 6, 2010

DOI: $10.3892 /$ ijo.2010.860

\begin{abstract}
Multidrug resistance (MDR) is a major reason for poor treatment results in hepatoblastoma (HB). The objective of this study was to establish a drug resistance model for HB to analyse alternative treatment options in vitro. Both $\mathrm{HB}$ cell lines HUH6 and HepT1 were xenotransplanted in NMRI mice (nu/nu) and 2 cycles of cisplatin (CDDP) treatment were administered. Thereafter, xenotransplants were excised and viable tumour cells were re-cultured. 3D cultures of HUH6 and HepT1 cells were generated on a low binding culture surface. Cell viability in response to $\mathrm{CDDP} / \mathrm{DOXO}$ (doxorubicin) and apoptosis was assessed by MTT-assay and caspase 3 activity, respectively. Efflux of doxorubicin was measured by flow cytometry. Cellular levels of ABC-transporters (MDR1, MRP1, cMOAT and BRCP) were determined by real time RTPCR. Only HepT1 cells isolated from HB xenografts showed resistance to CDDP, but did not survive repeated passages. Culturing HUH6 and HepT1 cells as spheroids was successful and $3 \mathrm{D}$ cultures showed an $\mathrm{IC}_{50}$-drift to higher drug concentrations for CDDP and DOXO compared to 2D cultures. Treatment with CDDP and DOXO led to homogeneous apoptosis in spheroids. Increased doxorubicin efflux in HUH6 spheroids was not influenced by the P-glycoprotein inhibitor tariquidar. Expression levels of MDR1, MRP1, cMOAT and BRCP in 3D cultures were similar to those in 2D cultures and were higher in HepT1 than in HUH6 cells. In conclusion, a 3D cell culture model for multidrug resistance was established for hepatoblastoma. The underlying mechanism involves altered accessibility of the cells for drugs rather than up-regulation of $\mathrm{ABC}$-transporters.
\end{abstract}

Correspondence to: Dr Sorin Armeanu-Ebinger, Department of Pediatric Surgery, University Children's Hospital, Hoppe-SeylerStrasse 1, 72076 Tuebingen, Germany

E-mail: sorin.armeanu-ebinger@med.uni-tuebingen.de

Abbreviations: HB, hepatoblastoma; CDDP, cisplatin; DOXO, doxorubicin; $\mathrm{IC}_{50}, 50 \%$ (cell viability) inhibiting concentration; MDR, multidrug resistance; PRETEXT, pretreatment extent of disease; ABC proteins, ATP-binding cassette proteins; MRP1, multidrug-resistance-associated protein 1; cMOAT, canalicular multispecific organic anion transporter, BCRP, breast cancer resistance protein

Key words: spheroid culture, drug resistance, hepatoblastoma

\section{Introduction}

Multidrug resistance (MDR) contributes to limited treatment results in human hepatoblastoma (HB) (1). While standard risk $\mathrm{HB}$ achieve a 3-year survival of nearly $95 \%$ through current treatment regimes including neoadjuvant chemotherapy and surgery, the outcome of patients with high risk or relapsed HB remains still poor with a 3-year survival of $69 \%$ (2-4). Treatment regimens are based on the results of different international trails combining perioperative chemotherapy with surgery (5-7). The International Childhood Liver Tumour Strategy Group (SIOPEL) developed the preoperative staging system PRETEXT (PRETreatment EXTent of disease) as a tool to classify patients in standard and high risk groups $(8,9)$. Pre- and postoperative chemotherapy regimens consist of different cycles of cisplatin and doxorubicin (PLADO) depending on the risk group. The most recent randomized trail, SIOPEL 3, revealed that cisplatin monotherapy achieves similar survival rates compared to cisplatin plus doxorubicin in children with standard risk hepatoblastoma (2). The regime with PLADO is still in use for patients in the high risk group.

Initially most HB tumours are chemosensitive; however they can acquire strategies to overcome drug therapy. Multidrug resistance has been identified as an important factor for diminished chemosensitivity $(10,11)$. A well known mechanism of chemoresistance is the ATP-dependent excretion of drugs from the tumour cell via ABC transporters (12).

Since the incidence of HB is relatively low, the establishment of experimental models became essential to investigate tumour biology, the phenomenon of drug resistance and possible new treatment options (13). Some continuous cell lines of $\mathrm{HB}$ are available for in vitro studies (14-16). To closer reflect the microenvironment of HB tumours, an animal model using nude mice (NMRI, nu/nu) was established (17). However, there exists no model to investigate the impact of substances on drug resistant HB. Creating drug resistant cells from pre-existing cell lines was successful in different tumours $(18,19)$. Furthermore, three-dimensional cell cultures have been established for human cancer cells. These so-called multicellular spheroids mimick solid tumours more closely than monolayer cells presenting an elevated resistance to chemotherapeutic agents $(20,21)$. Up to now no drug resistant hepatoblastoma model has been described. The aim of this study was to generate a model in order to analyse treatment options for chemoresistant $\mathrm{HB}$ in an experimental setting. 


\section{Materials and methods}

Cell culture. The continuous HB cell lines HepT1 and HUH6 were cultured on plastic culture dishes (Greiner, Essen, Germany) in Dulbecco's modified Eagle's medium (Biochrom, Berlin, Germany) supplemented with $10 \%$ fetal calf serum and $1 \%$ L-glutamine (Gibco, Eggenstein, Germany) at $37^{\circ} \mathrm{C}$ in a $95 \%$ air $/ 5 \% \mathrm{CO}_{2}$ humidified atmosphere. HUH6, first described by Doi, derived from a mixed HB (14). HepT1 originated from a multifocal embryonal HB (15). For spheroid cultures low attachment plates were used (Corning Inc., Corning, NY, USA). Explanted tumour cells from previously xenotransplanted HB were re-cultured and supplemented with penicillin/streptomycin (100 units/ml). After 2-3 cell cycles xenograft-derived HB cells were used for further experiments (Fig. 1A).

Animals and xenotransplantation. For the generation of cisplatin resistant tumour cells we used a previously described animal model $(17,22)$. HUH6 and HepT1 cells were xenotransplanted into 6 to 8 -week-old, female athymic NMRI (nu/nu) mice. All animal studies were approved by the local government's ethics authority of Tuebingen (No. CK 1/08). Animals were kept under pathogen-free conditions and were fed ad libitum with autoclaved food and sterilized water. For each tumour, $1 \mathrm{ml}$ of tumour cell suspension (approximately 3 million cells) was injected into paravertebral areas subcutaneously. At a tumour size of approximately $20 \mathrm{~mm}^{3}$, mice were treated with cisplatin (CDDP, $3 \mathrm{mg} / \mathrm{kg}$ ) intraperitoneally at days 1-3 and 14-16. Animals were sacrificed at day 20 . Tumours were explanted, mechanically isolated and xenograftderived HB cells were re-cultured. Parts of the tumour were processed for H\&E staining (16).

Cell viability. Cell viability was assessed by MTT [3-(4,5dimethylthiazol-2-yl)-2,5-diphenyl-tetrazoliumbromide]-assay (EZ4U, Biomedica, Vienna, Austria). HB cells and xenograft derived HB cells were cultured under afore-said conditions in 96-well plates $\left(10^{4}\right.$ cells/100 $\left.\mu 1\right)$ (Becton-Dickinson $\mathrm{GmbH}$, Heidelberg, Germany). After $24 \mathrm{~h}$ CDDP (0.1-10.0 $\mu \mathrm{M})$ or DOXO (0.01-10.0 $\mu \mathrm{M})$ were added. Drug dilutions were prepared immediately before administration. Incubation lasted for $72 \mathrm{~h}$. All assays were performed 3 times in quadruplicates. MTT assay was carried out according to the manufacturer's protocol. Substrate was dissolved in activator solution and $20 \mu \mathrm{l}$ were added to each well. Incubation lasted for $1 \mathrm{~h}$ and absorption was then measured using a Milenia Kinetic Analyzer (DPC Biermann, Bad Nauheim, Germany) at $450 \mathrm{~nm}$. Percentages of cell viability were calculated by normalization between background of cultures without cells and untreated cultures as control experiments.

Apoptosis assay. Apoptosis was monitored using the cleavage of the fluorogenic DNA-binding dye from a peptide with caspase 3 recognition sequence. 3D cultures were either treated with CDDP $(1.25 \mu \mathrm{g} / \mathrm{ml})$ or DOXO $(0.1 \mu \mathrm{g} / \mathrm{ml})$ for 48 h. DEVD-NucView 488 (Biotrend, Koeln, Germany) was then added to the culture medium to a final concentration of $5 \mu \mathrm{M}$. Activated caspase 3 cleaves DEVD-NucView 488 delivering the green fluorescent dye. Apoptotic cells were detected after 90 min using a Zeiss Axiophot epifluorescence microscope and the AxioVision software (Carl Zeiss, Oberkochen, Germany).

Dye exclusion assay. HB cells were cultured for $72 \mathrm{~h}$ as monolayers or $3 \mathrm{D}$ cultures. Cells were then harvested by trypsination and incubated for $30 \mathrm{~min}$ at $37^{\circ} \mathrm{C}$ in the dark in DMEM medium supplemented with $50 \mu \mathrm{M}$ DOXO. Cell suspension was washed twice in cold PBS and split into 3 tubes. Efflux samples were incubated for $60 \mathrm{~min}$ in DMEM medium without DOXO at $37^{\circ} \mathrm{C}$. Non-efflux samples were maintained at $4^{\circ} \mathrm{C}$ until FACS analysis. In some experiments, the P-glycoprotein inhibitor tariquidar (XR9576, Millennium Pharmaceuticals Inc. Cambridge, MA, USA) was added during the efflux incubation in a concentration of $2.5 \mu \mathrm{M}$. Fluorescence intensity in channel 4 from FACScalibur (BD Biosciences, Heidelberg, Germany) was compared. Efflux activity was estimated as the proportion of cells with lower fluorescence intensity than in non-efflux samples.

Quantitative real-time reverse transcription PCR (Qrt-RT$P C R)$. Messenger RNA levels of four drug-transporters (BCRP/ABCG2, cMOAT/ABCC2, MRP1/ABCC1; MDR1/ ABCB1; Applied Biosystems, Darmstadt, Germany) were determined in triplicates from HUH6 and HepT1 cells either grown as monolayer or 3D culture. Isolation of total RNA was performed with Quiagen RNeasy Mini kit (Qiagen, Hilden, Germany) according to manufacturer's protocol. Reverse transcription was performed using a high capacity cDNA archive kit and $1 \mu \mathrm{g}$ RNA into $20 \mu \mathrm{l}$ assay (Applied Biosystems, Darmstadt, Germany). The gene expression level of the drug transporters was measured by TaqMan ${ }^{\mathrm{TM}}$ detection via quantitative real-time reverse transcription PCR. The reactions were performed in MicroAmp ${ }^{\mathrm{TM}}$ optical 96-well reaction plates (Applied Biosystems, Darmstadt, Germany) and incubated for $2 \mathrm{~min}$ at $50^{\circ} \mathrm{C}$ for 1 cycle, for $10 \mathrm{~min}$ at $95^{\circ} \mathrm{C}$ for 1 cycle, for $15 \mathrm{sec}$ at $95^{\circ} \mathrm{C}$ for 1 cycle and for $1 \mathrm{~min}$ at $60^{\circ} \mathrm{C}$ for 40 cycles using CFX96 ${ }^{\mathrm{TM}}$ Real-Time System (BioRad Laboratories, Munich, Germany). The following TaqMan gene expression assays (Applied Biosystems) were used: i) breast cancer resistance protein (BCRP, Hs01053787_m1), ii) canalicular multispecific organic anion transporter (cMOAT, Hs00166123_m1), iii) multidrug resistance gene 1 (MDR1, s00184491_m1), iv) MDR-associated protein 1 (MRP1, Hs00219905_m1) and v) pyruvate dehydrogenase (lipoamide) B (PDHB, Hs00168650_m1) served as control. Probes were labelled with the fluorescent dye 5-carboxyfluoroscein (FAM). Relative expression levels were calculated using the $\Delta$ threshold cycle-method $(\Delta \mathrm{CT})$ with PDHB as control. Differences in gene expression were calculated by the $\Delta \Delta$ threshold cycle-method $(\Delta \Delta \mathrm{CT})$ using the corresponding monolayer cultures as reference sample.

Statistical analysis. Statistical analysis was carried out by one way ANOVA on ranks test using GraphPad Prism 4.00 (GraphPad Software, San Diego, CA, USA). Viability curves were fitted with a sigmoidal dose response function with variable slope. All numeric data are expressed as mean $\pm \mathrm{SD}$ or $95 \%$ confidence interval. Significance was assumed for all results at $<0.05$. 


\section{Results}

Generating cisplatin resistant HepT1 cells using a xenograft model. The first approach in generating drug resistant $\mathrm{HB}$ tumour cells was to escalate drug concentration in $\mathrm{HB}$ cell cultures. Adding CDDP (HUH6: $0.4 \mu \mathrm{g} / \mathrm{ml}$, HepT1: 1.0 $\mu \mathrm{g} / \mathrm{ml}$ ) or DOXO (HUH6: $0.02 \mu \mathrm{g} / \mathrm{ml}$, HepT1: $0.9 \mu \mathrm{g} / \mathrm{ml}$ ) to HB cell cultures resulted in more than $50 \%$ of viable cells after $72 \mathrm{~h}$ of culturing. However, cells died after the first passages at this low concentrations and it was not possible to further escalate the drug concentrations. Previous experiments have demonstrated a survival of HB cells in xenografts after treatment with CDDP (1). Thus we questioned if these cells might be drug resistant and could be re-cultured. We therefore used a xenograft animal model applying CDDP (3 mg/kg) in NMRI mice with xenotransplanted HB tumours (Fig. 1A). Chemotherapy led to reduced tumour growth in xenotransplanted HB tumours of both cell lines as described previously (23). However, the explanted tumours contained among necrotic regions, viable tumour cells as shown by cell integrity in $\mathrm{H} \& \mathrm{E}$ histological staining (Fig. 1B). Xenograft derived HB cells could be re-cultured after tumour explantation and MTT assay was performed to determine cell viability under CDDP treatment. Treatment with CDDP revealed a shift to higher $\mathrm{IC}_{50}$ concentrations for xenograft derived HepT1 cells compared to parental HepT1 cells $\left(\mathrm{IC}_{50} 4.6 \mu \mathrm{g} / \mathrm{ml} \pm 0.2\right.$ to $8.4 \mu \mathrm{g} / \mathrm{ml} \pm 0.1$ ) (Fig. 1C). Xenograft derived HUH6 cells showed no change in viabillity in comparison to the HUH6 continuous cell line (data not shown). Repeating cell passages as well as freezing and thawing cycles for further experiments were not successful.

Cell viability in $H B$ spheroid cultures. Through culturing of $\mathrm{HB}$ cells on low binding plates, spheroids were formed with a mean spheroid diameter of $953.9 \pm 453.1 \mu \mathrm{m}$ for HUH6 and $1085.0 \pm 319.8 \mu \mathrm{m}$ for HepT1. 3D cultures of the pediatric $\mathrm{HB} / \mathrm{HCC}$ cell line HepG2 have been reported to show a diminished sensitivity to cytostatics (21). We therefore tested the viability of HUH6 and HepT1 cells grown as 3D cultures in comparison with 2D cultures. Cell viability declined with increasing concentrations of CDDP. The $\mathrm{IC}_{50}$ for HUH6 cells was $2.4 \mu \mathrm{g} / \mathrm{ml}(95 \%$ CI 1.8-3.3) and for HepT1 $4.1 \mu \mathrm{g} / \mathrm{ml}$ (95\% CI 1.5-12.4) when grown as monolayer (Fig. 2A and C). Culturing HepT1 cells as spheroids the concentrations of CDDP to reach $50 \%$ growth inhibition was elevated to $6.75 \mu \mathrm{g} / \mathrm{ml}$. A concentration of $10 \mu \mathrm{g} / \mathrm{ml} \mathrm{CDDP}$ did not lead to a growth inhibition of more than $80 \%$ in HUH6 spheroids. Treatment with DOXO showed a similar effect. In 2D cultures $50 \%$ growth inhibition was reached at $0.2 \mu \mathrm{g} / \mathrm{ml}$ (95\% CI 0.1-0.2) for HUH6 cells and $1.3 \mu \mathrm{g} / \mathrm{ml}$ (95\% CI 0.8-2.3) for HepT1 cells (Fig. 2B and D). At the same concentrations of DOXO the cell viability in $3 \mathrm{D}$ cultures only decreased to $75 \%$ in HUH6 cells and 80\% in HepT1 cells, respectively.

Induction of apoptosis in HB spheroid cultures. To further investigate the effect of $3 \mathrm{D}$ culturing on the viability of $\mathrm{HB}$ cells we monitored the activation of caspase 3 (Fig. 3). In control spheroid cultures apoptosis was detectable at low levels. Incubating 3D cultures of HUH6 and HepT1 with CDDP $(1.25 \mu \mathrm{g} / \mathrm{ml})$ or DOXO $(0.1 \mu \mathrm{g} / \mathrm{ml})$ led to enhanced
A

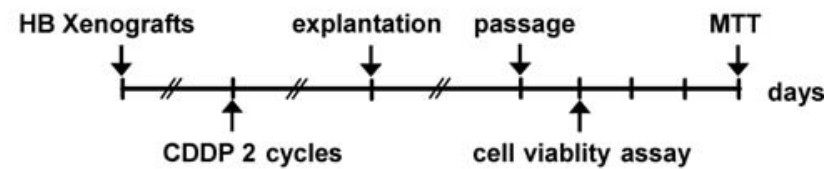

B

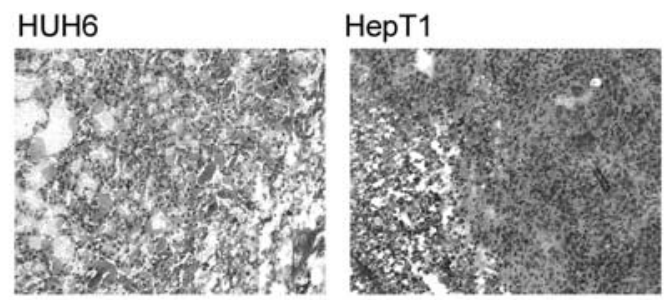

C

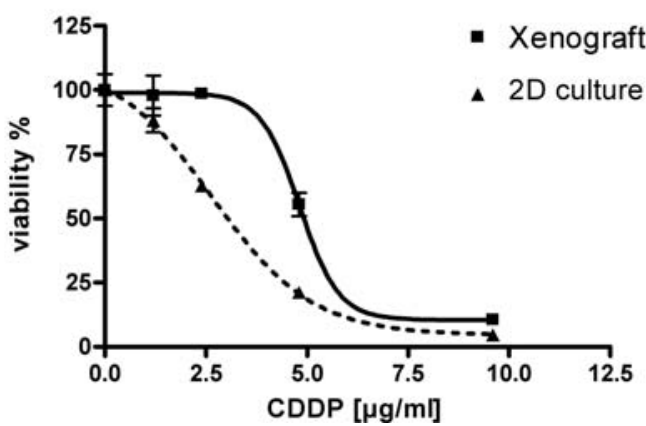

Figure 1. (A) Flowchart of animal model for selection of CDDP resistant HB cells: HB tumours were grown as xenografts in NMRI (nu/nu) mice. After 2 cycles of CDDP treatment tumour cells were explanted and recultured. Cells were used for MTT assays after 2-3 passages. (B) H\&E staining of explanted tumours: regions of necrotic and viable cells were detected in both xenografted tumours (HUH6 and HepT1) after CDDP treatment. (C) Response of xenograft derived HepT1 cells to CDDP: cells derived from xenografts and $2 \mathrm{D}$ cultured cells were incubated with CDDP for $72 \mathrm{~h}$ and cell viability was determined by MTT assay compared to untreated cells. Data represent mean \pm SD of relative cell viability from triplicates.

apoptosis rates. Distribution of apoptotic cells was homogeneous over the whole spheroids, there was no central necrotic zone detectable. Thus CDDP and DOXO exerted apoptosis in both HB cell lines cultured as spheroids.

Activity of drug transporters in $H B$ cells. To address the mechanism of diminished chemosensitivity of HB cells cultured as spheroids a functional test of doxorubicin efflux was performed. Single cell suspensions from differentially cultured HB cells were incubated with doxorubicin and the efflux of the red fluorescent drug was measured by flow cytometry. HUH6 cells derived from spheroids accumulate doxorubicin to lower extent than those from $2 \mathrm{D}$ cultures as revealed by the portion of cells in the right upper quadrant of $70 \%$ and $97 \%$, respectively (Fig. 4, mid column). There was no difference in the doxorubicin load between HepT1 3D and $2 \mathrm{D}$ cultures. The efflux of doxorubicin was calculated as percentage of cells with low fluorescence after incubating the cells at $37^{\circ} \mathrm{C}$ for $60 \mathrm{~min}$ (Fig. 4, left column). The intracellular doxorubicin concentration was decreased in $83 \%$ of the cells from HUH6 3D cultures. In contrast no drug efflux was detected in 2D cultures of HUH6 (1\%). There was a doxorubicin efflux in both HepT1 3D and 2D cells, independent of the culture conditions (2D: 10\%, 3D: 14\%). To 

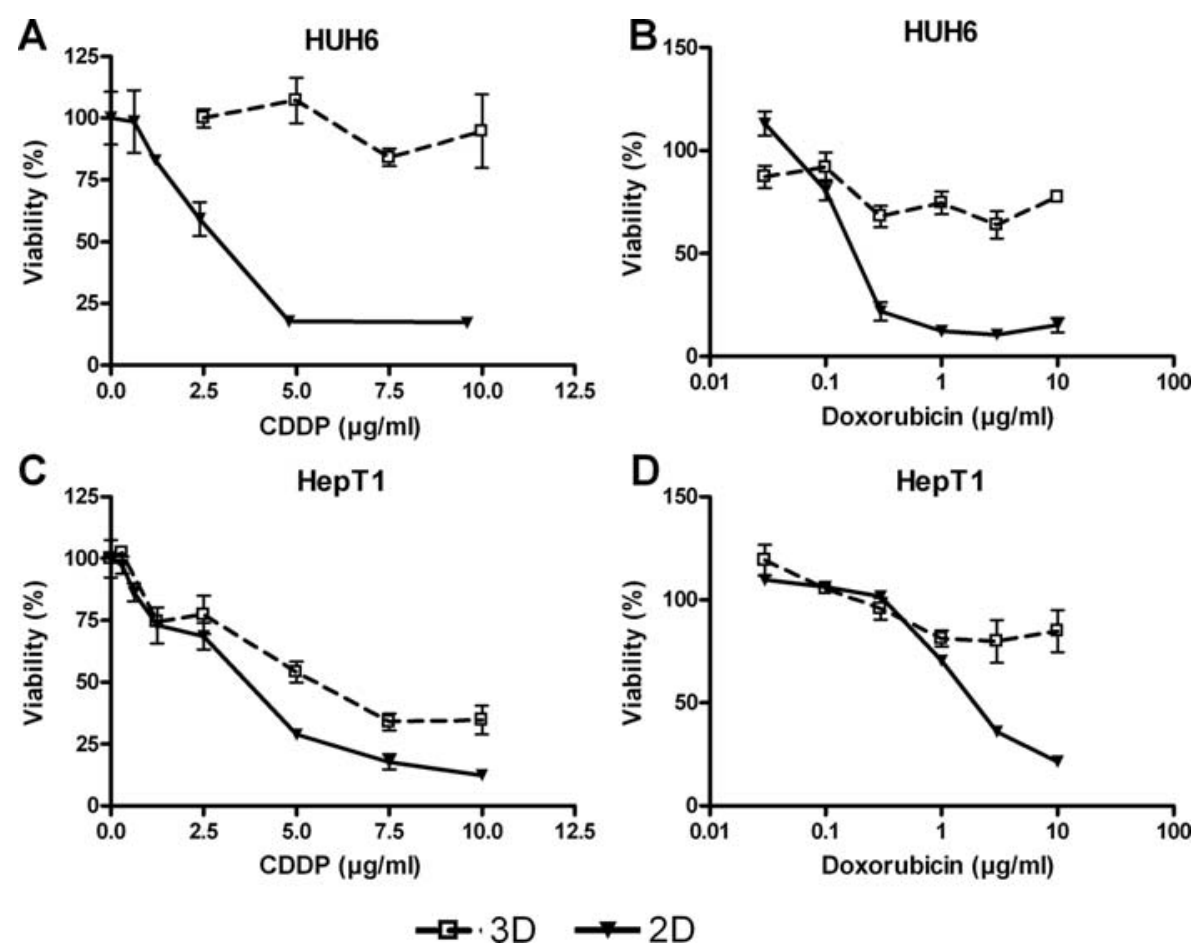

Figure 2. Effect of CDDP and DOXO on HB cell viability: HUH6 and HepT1 were cultured as monolayer (2D) or as spheroids (3D). Cultures were treated with increasing concentrations of CDDP and DOXO. Cell viability was related to untreated control cultures. Data represent mean \pm SD from triplicates.

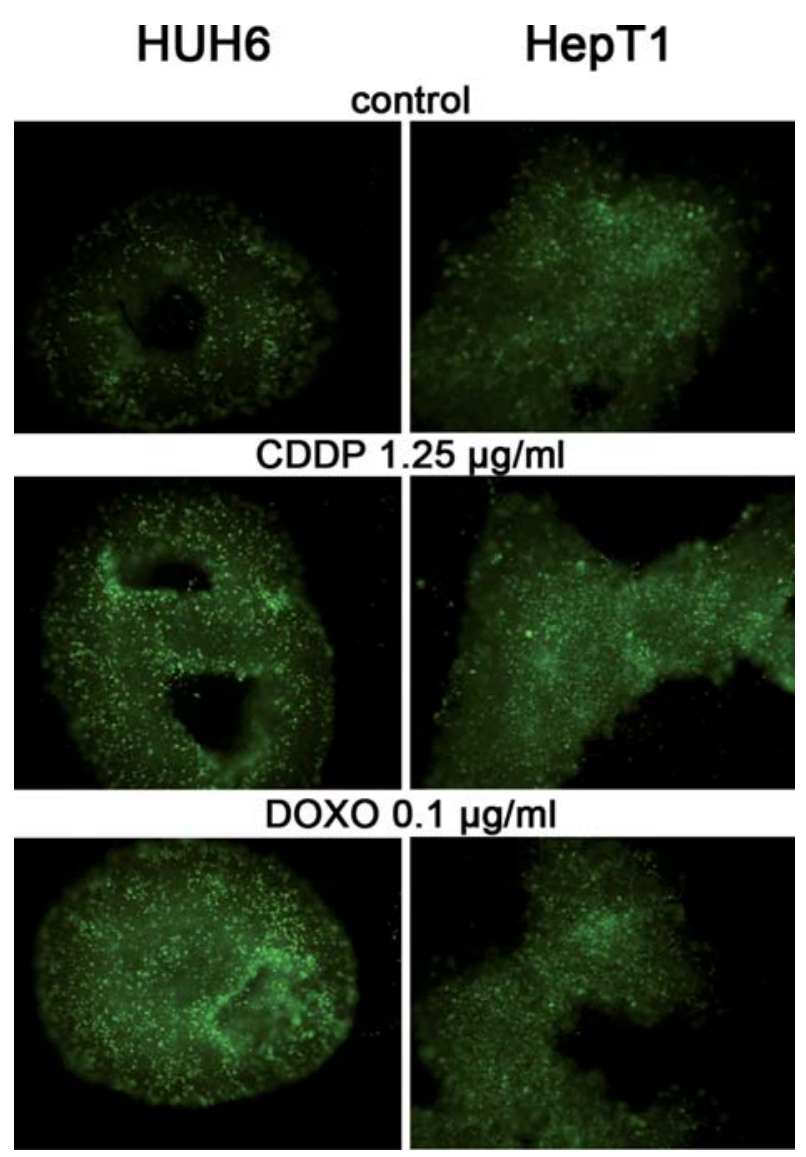

Figure 3. Induction of apoptosis in HB cells cultured as spheroids: 3D cultures of HUH6 and HepT1 were treated with CDDP and DOXO for $48 \mathrm{~h}$ Caspase 3 activation was detected with the NucView-488 substrate. Green fluorescent cells denote apoptotic cells (microscope magnification x5). further investigate the mechanism of doxorubicin release, cells were incubated with tariquidar (XR 9576), an inhibitor of P-glycoprotein (MDR1, ABCB1). As illustrated in the right lateral column, inhibition of $\mathrm{P}$-glycoprotein did not alter the doxorubicin efflux in HUH6 and HepT1 spheroid cells. Neither the doxorubicin uptake nor the efflux was influenced by tariquidar in HUH6 2D cultures. In contrast there was a slight inhibition of doxorubicin efflux by tariquidar in HepT1 2D cultured cells (1\% vs. 10\%). Flow cytometry analysis revealed a shift of the cells to lower values of forward light scatter when cultured as spheroids, which denotes a smaller cell volume compared to cells grown as monolayer. In summary, efflux of doxorubicin seems not to be dependent on MDR1 in this culture system.

Expression of MDR-related genes in HB spheroids. HB cells cultured as spheroids showed drug resistance to CDDP and DOXO. The phenotype of multidrug resistance has been linked to altered expression of several genes $(12,24)$. Therefore we investigated, whether spheroids of HB cells show a changed expression of MDR related genes compared to 2D cultures. BCRP, cMOAT, MDR1 and MRP1 were quantified by real-time reverse transcription-PCR. The relative expression of these genes is demonstrated in Fig. 5. Transcripts for all analysed genes were detected in both cell lines. cMOAT and MDR1 were the most prominently expressed genes in HepT1 cells. In HUH6 cells BCRP and MRP1 were higher expressed than cMOAT and MDR1 (Fig. 5A). Relating the expression levels of both cell lines for each tested gene, there was a more than 125-fold higher expression of cMOAT and a more than 900-fold higher expression of MDR1 in HepT1 cells compared to HUH6 cells, independent of the culture 
A
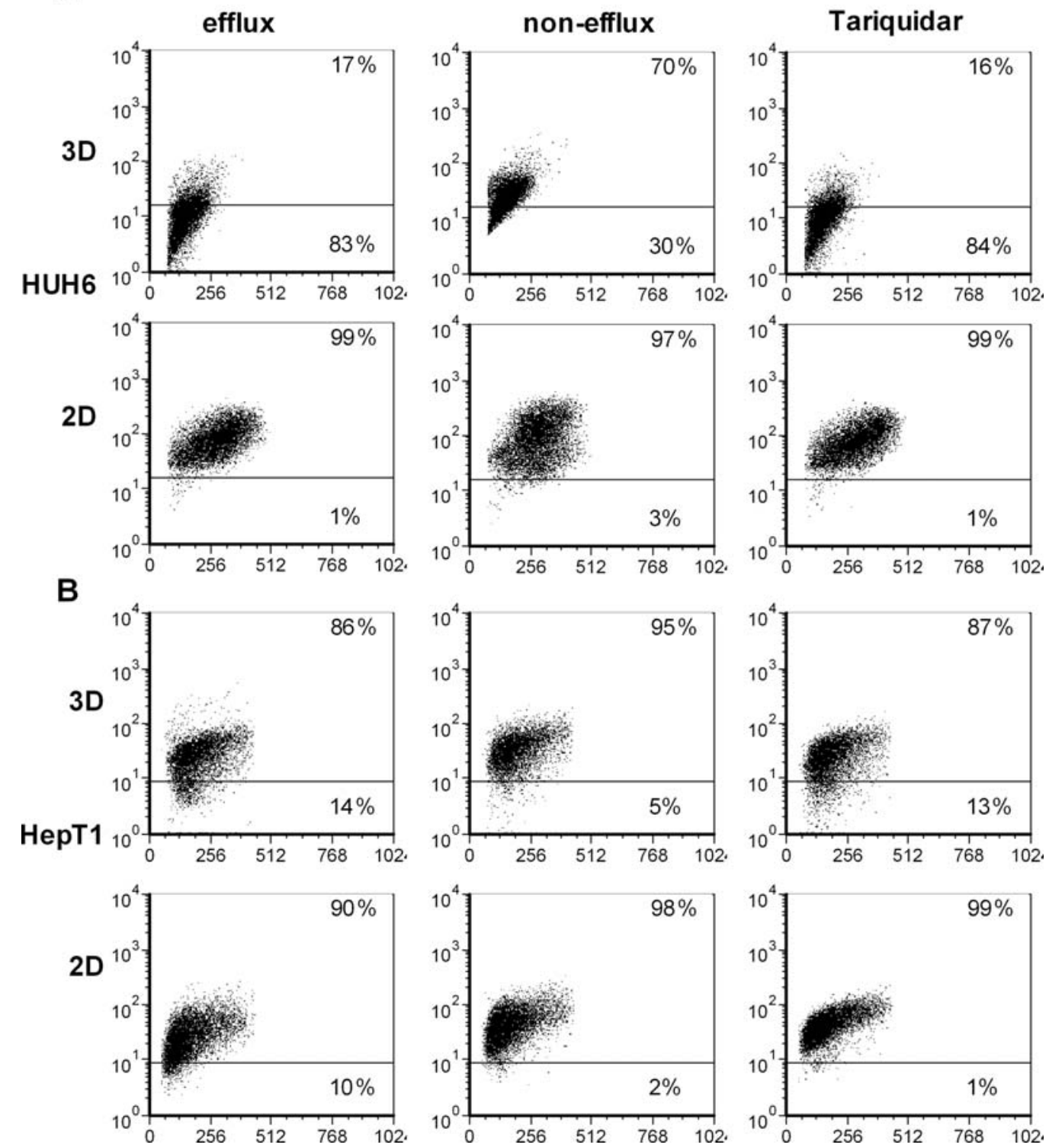

Figure 4. Uptake and efflux of doxorubicin in HB cells: HUH6 (A) and HepT1 (B) were cultured on plate surface (2D) or as spheroids (3D). Cells were loaded with DOXO. Fluorescence of DOXO in HB cells was detected by flow cytometry post incubation at growing conditions (efflux) and at the time of cell-loading (non-efflux). Tariquidar was used as inhibitor of P-glycoprotein (MDR1). The percentages given in the right lower corner denote the amount of cells which removed DOXO.

conditions (Fig. 5B). Comparing the gene expression of both cell culture conditions for each cell line, there was a significant 2.4-fold lower expression of cMOAT in HepT1 cells when cultured as spheroids compared to $2 \mathrm{D}$ cultures $(\mathrm{p}<0.05$, ANOVA test), while expression of BCRP, MDR1 and MRP1 remained unchanged in both cell lines (Fig. 5C and D).

\section{Discussion}

Drug resistance is a major reason for poor treatment results in high risk and recurrent hepatoblastoma (1). Multicenter studies have evidenced multidrug resistance (MDR) occurring in up to $80 \%$ of hepatoblastoma tumours after 4 or 5 cycles of chemotherapy (11). A model reflecting the conditions of chemoresistant hepatoblastoma to assess alternative treatment strategies preclinically is thus highly desirable.
Drug resistance can be divided in intrinsic, which can be pre-existing or acquired, and extrinsic resistance. Intrinsic mechanisms of drug resistance are various: induction of efflux transport proteins (ABC proteins, P-glycoprotein), enhancement of DNA repair, maintenance of cells in a predominantly non-cycling state, protection from induced apoptosis and overexpression of detoxification systems, like glutathione transferases $(12,25-28)$. In contrast to those cellular mechanisms the extrinsic resistance is dependent on tumour structure, with regions of hypoxia and acidosis, on vascularisation due to the release of pro-angiogenic factors and nutrition supply (29).

We tested different approaches to obtain chemoresistant HB cells. The only successful way to achieve resistance in vitro was to culture HB cells as spheroids. In our setting the use of increasing dosages of anticancer agents on pre-existing cell 

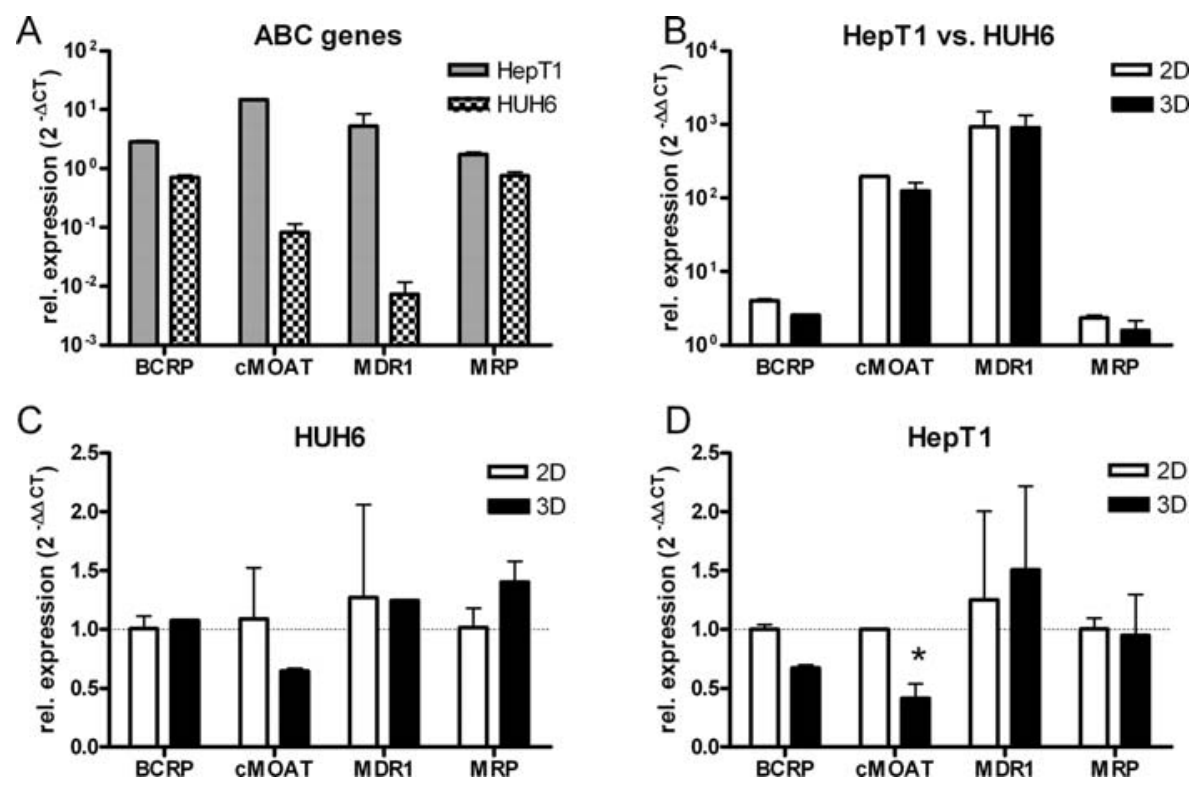

Figure 5. Expression of MDR related genes in HB cells. HUH6 and HepT1 were cultured on plate surface (2D) or as spheroids (3D). mRNA of BCRP, cMOAT, MDR1 and MRP was quantified by RT-PCR. Gene expression was related to PDHB as housekeeping gene (A). Relative expression of genes was compared by the $\triangle \triangle \mathrm{CT}$ method, HUH6 served as reference cell line (B). 3D cultures were compared to 2D cultures for HUH6 (C) and HepT1 (D). Data represent the mean \pm SD from two biological replicates. Significant differential gene expression is indicated (" $\mathrm{p}<0.05$, Anova test).

lines (HUH6, HepT1) was not applicable. This approach has been previously described for other tumours $(18,19)$. After treatment with CDDP the selected HB cells did not survive repeated cell passages. This might be caused by an altered adhesion capability of the cells to the surface of culture plates. CDDP is known to increase fluidity of cell membranes through oxidation. Accordingly, we observed a destabilization of cell integrity in both tested cell lines together with an altered nucleus-cytoplasm relation on the basis of increased numbers of nuclei $(30,31)$. An animal model with exposure to CDDP was used to overcome the problem of reduced cell-cell adhesions (17). After re-culturing, xenograft derived HB cells from HepT1, but not from HUH6, showed an enhanced $\mathrm{IC}_{50}$ for CDDP. The increased disposition of HepT1 cells to acquire chemoresistance might be explained by their origin. HepT1 cells are derived from an embryonal tumour and exhibit a less differentiation compared to HUH6 cells which originate from a mixed HB $(14,15)$. However, as cryopreservation of these cells failed no stable CDDP-resistant hepatoblastoma cell line could be established using this procedure.

Spheroids represent a system in which tumour cells are growing in a three-dimensional fashion with tight cell-cell interactions. Previous reports described the successful establishment of 3D cultures for different tumours following the idea of mimicking the biological microenvironment of tumour cells including intrinsic and extrinsic mechanism of resistance $(20,21,32)$. In these studies the morphology of spheroids and the dose response curves to anticancer drugs were close to experimental tumours in vivo (19). However, the influence of tumour vascularisation and possible interactions with the host immune system cannot be considered. Spheroid cultures of HB cell lines were feasible and showed tight cellcell adhesions as trypsination was necessary to break the 3D structure. Spheroids of HB cells presented larger diameters compared to previous reports of 3D cultures of HepG2 cells and lung cancer cells $(21,33)$. In contrast to several descriptions there was no heterogeneity or central necrotic zone as apoptosis occurred homogeneously in HB 3D cultures (20). The missing central necrotic zone in our setting might be caused by a less pronounced cell-cell interaction providing sufficient nutrition of cells in the center of the spheroids. Resistance to cisplatin and doxorubicin in spheroids of both cell lines was evidenced by a drift to higher $\mathrm{IC}_{50}$ concentrations compared to two-dimensional cultured cells. This observation is possibly resulting from decreased concentrations of anticancer drugs within the cells caused by the cell arrangements and expression of $\mathrm{ABC}$ transporters in $3 \mathrm{D}$ cultures. As a model for drug transport using doxorubicin spheroids of HUH6 and HepT1 showed an enhanced drug efflux, while there was no efflux in monolayer cultures of HUH6. Doxorubicin efflux could not be influenced through the P-glycoprotein inhibitor tariquidar. The same observation has been made in spheroids of human lung cancer cells which retained 3-fold lower doxorubicin concentrations compared to monolayer cultures, independently of inhibition of P-glycoprotein by cyclosporine A and ATP (33). However, as HB cells express MDR, inhibition of P-glycoprotein in monolayer cultures is successful when cells are constantly exposed to the inhibitors, such as PSC833 $(22,23)$.

Like P-glycoprotein overexpression of $\mathrm{ABC}$ genes in chemotherapy-resistant tumours and up-regulation after chemotherapy has been observed in various paediatric tumours $(12,34)$. In hepatoblastoma different studies revealed an upregulation of MDR1 and BCRP mRNA expression after standard chemotherapy with cisplatin and doxorubicin $(1,24,35)$.

We found the highest expression of MDR1 and cMOAT in HepT1 cells. This is consistent with previous studies 
describing overexpression of MDR1 and cMOAT in HB cell lines and in patients with embryonal HB $(24,36)$. However, a recent study investigating expression levels in 7 patients with hepatoblastoma found an increased expression of BCRP after chemotherapy while the levels of MDR1, MRP1 and cMOAT remained unchanged (35). Interestingly, none of the analysed tumour samples contained embryonal components, which is a known phenomenon after chemotherapy of HB tumours (11). In HUH6 cells the BCRP and MRP1 genes showed the highest expression levels. Hence different expression of ABC genes might be associated with the different histological subtypes of HB tumours. Expression levels of cMOAT were lower in HB spheroids compared to monolayer cultures, which might be caused by a decreased cell volume and increased cell-cell adhesion in spheroids. Overexpression of cMOAT was described for other human cancer cell lines as result of CDDP treatment, a setting which did not correspond with the emerging resistance in our spheroid cultures, without CDDP challenge (37). Thus, the observed heterogeneous expression of $\mathrm{ABC}$ transporters does not support our hypothesis of upregulation of drug transporters in HB spheroids.

While culturing cells in spheroids more closely mimics tumour growth in vivo, the mechanism of resistance may be of mechanical, structural or cell biological reasons. The increased $\mathrm{IC}_{50}$, lower drug load and enhanced drug excretion observed in our model might be mediated by an increased activity of $\mathrm{ABC}$ transporters rather than an up-regulated expression. Drug resistance could also evolve due to the drug concentration gradient occurring in 3D structures, however the homogeneous apoptosis observed in spheroids supports an intrinsic cell resistance $(20,33)$.

Taken together we established a chemoresistance model for $\mathrm{HB}$ using $3 \mathrm{D}$ cell cultures. This model might serve as a tool for investigating various biological aspects in this malignancy, such as drug resistance, in which drug access to cells and cell-cell contact limit the treatment efficiency. Selecting efficient drugs in this three-dimensional hepatoblastoma culture system may expedite drug screening for alternative treatment options of HB.

\section{Acknowledgements}

This study was supported by the research funding programme of the Faculty of Medicine, University of Tuebingen (fortüne 1830-0-0). The authors declare no conflict of interest related to this study.

\section{References}

1. Warmann S, Hunger M, Teichmann B, Flemming P, Gratz KF and Fuchs J: The role of the MDR1 gene in the development of multidrug resistance in human hepatoblastoma: clinical course and in vivo model. Cancer 95: 1795-1801, 2002.

2. Perilongo G, Maibach R, Shafford E, et al: Cisplatin versus cisplatin plus doxorubicin for standard-risk hepatoblastoma. N Engl J Med 361: 1662-1670, 2009.

3. Fuchs J, Rydzynski J, Von Schweinitz D, et al: Pretreatment prognostic factors and treatment results in children with hepatoblastoma: a report from the German Cooperative Pediatric Liver Tumor Study HB 94. Cancer 95: 172-182, 2002.

4. Zsiros J, Maibach R, Shafford E, et al: Successful treatment of childhood high-risk hepatoblastoma with dose-intensive multiagent chemotherapy and surgery: final results of the SIOPEL3HR study. J Clin Oncol 28: 2584-2590, 2010.
5. Ortega JA, Douglass EC, Feusner JH, et al: Randomized comparison of cisplatin/vincristine/fluorouracil and cisplatin/ continuous infusion doxorubicin for treatment of pediatric hepatoblastoma: A report from the Children's Cancer Group and the Pediatric Oncology Group. J Clin Oncol 18: 2665-2675, 2000.

6. Perilongo G, Shafford E and Plaschkes J: SIOPEL trials using preoperative chemotherapy in hepatoblastoma. Lancet Oncol 1: 94-100, 2000

7. Sasaki F, Matsunaga T, Iwafuchi M, et al: Outcome of hepatoblastoma treated with the JPLT-1 (Japanese Study Group for Pediatric Liver Tumor) Protocol-1: A report from the Japanese Study Group for Pediatric Liver Tumor. J Pediatr Surg 37: 851-856, 2002.

8. Brown J, Perilongo G, Shafford E, et al: Pretreatment prognostic factors for children with hepatoblastoma - results from the International Society of Paediatric Oncology (SIOP) study SIOPEL 1. Eur J Cancer 36: 1418-1425, 2000.

9. Perilongo G, Shafford E, Maibach R, et al: Risk-adapted treatment for childhood hepatoblastoma. Final report of the second study of the International Society of Paediatric OncologySIOPEL 2. Eur J Cancer 40: 411-421, 2004.

10. Von Schweinitz D, Hecker H, Harms D, et al: Complete resection before development of drug resistance is essential for survival from advanced hepatoblastoma - a report from the German Cooperative Pediatric Liver Tumor Study HB-89. J Pediatr Surg 30: 845-852, 1995

11. Von Schweinitz D, Byrd DJ, Hecker H, et al: Efficiency and toxicity of ifosfamide, cisplatin and doxorubicin in the treatment of childhood hepatoblastoma. Study Committee of the Cooperative Paediatric Liver Tumour Study HB89 of the German Society for Paediatric Oncology and Haematology. Eur J Cancer 33: 1243-1249, 1997.

12. Leonard GD, Fojo T and Bates SE: The role of ABC transporters in clinical practice. Oncologist 8: 411-424, 2003.

13. Ishak KG and Glunz PR: Hepatoblastoma and hepatocarcinoma in infancy and childhood. Report of 47 cases. Cancer 20: 396-422, 1967.

14. Doi I: Establishment of a cell line and its clonal sublines from a patient with hepatoblastoma. Gann 67: 1-10, 1976.

15. Pietsch T, Fonatsch C, Albrecht S, Maschek H, Wolf HK and von Schweinitz D: Characterization of the continuous cell line HepT1 derived from a human hepatoblastoma. Lab Invest 74: 809-818, 1996.

16. Chen TT, Rakheja D, Hung JY, et al: Establishment and characterization of a cancer cell line derived from an aggressive childhood liver tumor. Pediatr Blood Cancer 53: 1040-1047, 2009.

17. Fuchs J, Schmidt D, Pietsch T, Miller K and von Schweinitz D: Successful transplantation of human hepatoblastoma into immunodeficient mice. J Pediatr Surg 31: 1241-1246, 1996.

18. Cocker HA, Tiffin N, Pritchard-Jones K, Pinkerton CR and Kelland LR: In vitro prevention of the emergence of multidrug resistance in a pediatric rhabdomyosarcoma cell line. Clin Cancer Res 7: 3193-3198, 2001.

19. Hong WS, Saijo N, Sasaki Y, et al: Establishment and characterization of cisplatin-resistant sublines of human lung cancer cell lines. Int J Cancer 41: 462-467, 1988.

20. Desoize B, Gimonet D and Jardiller JC: Cell culture as spheroids: an approach to multicellular resistance. Anticancer Res 18: 4147-4158, 1998.

21. Li CL, Tian T, Nan KJ, et al: Survival advantages of multicellular spheroids vs. monolayers of HepG2 cells in vitro. Oncol Rep 20: 1465-1471, 2008.

22. Warmann S, Gohring G, Teichmann B, Geerlings H, Pietsch T and Fuchs J: P-glycoprotein modulation improves in vitro chemosensitivity in malignant pediatric liver tumors. Anticancer Res 23: 4607-4611, 2003.

23. Warmann SW, Heitmann H, Teichmann B, et al: Effects of Pglycoprotein modulation on the chemotherapy of xenotransplanted human hepatoblastoma. Pediatr Hematol Oncol 22: 373-386, 2005.

24. Bader P, Fuchs J, Wenderoth M, von Schweinitz D, Niethammer D and Beck JF: Altered expression of resistance associated genes in hepatoblastoma xenografts incorporated into mice following treatment with adriamycin or cisplatin. Anticancer Res 18: 3127-3132, 1998.

25. Chu G: Cellular responses to cisplatin. The roles of DNAbinding proteins and DNA repair. J Biol Chem 269: 787-790, 1994. 
26. Fulda $\mathrm{S}$ and Debatin KM: Extrinsic versus intrinsic apoptosis pathways in anticancer chemotherapy. Oncogene 25: 4798-4811, 2006.

27. Szakacs G, Paterson JK, Ludwig JA, Booth-Genthe C and Gottesman MM: Targeting multidrug resistance in cancer. Nat Rev Drug Discov 5: 219-234, 2006.

28. Sau A, Pellizzari Tregno F, Valentino F, Federici G and Caccuri AM: Glutathione transferases and development of new principles to overcome drug resistance. Arch Biochem Biophys 500: 116-122, 2010.

29. Teicher BA: Acute and chronic in vivo therapeutic resistance. Biochem Pharmacol 77: 1665-1673, 2009.

30. Rebillard A, Lagadic-Gossmann D and Dimanche-Boitrel MT: Cisplatin cytotoxicity: DNA and plasma membrane targets. Curr Med Chem 15: 2656-2663, 2008.

31. Liang $X$ and Huang Y: Alteration of membrane lipid biophysical properties and resistance of human lung adenocarcinoma A(549) cells to cisplatin. Sci China C Life Sci 44: 25-32, 2001.

32. Kolchinsky A and Roninson IB: Drug resistance conferred by MDR1 expression in spheroids formed by glioblastoma cell lines. Anticancer Res 17: 3321-3327, 1997.
33. Ponce de Leon V and Barrera-Rodriguez R: Changes in Pglycoprotein activity are mediated by the growth of a tumour cell line as multicellular spheroids. Cancer Cell Int 5: 20, 2005.

34. Oue T, Yoneda A, Uehara S, Yamanaka H and Fukuzawa M: Increased expression of multidrug resistance-associated genes after chemotherapy in pediatric solid malignancies. J Pediatr Surg 44: 377-380, 2009.

35. Vander Borght S, van Pelt J, van Malenstein $\mathrm{H}$, et al: Upregulation of breast cancer resistance protein expression in hepatoblastoma following chemotherapy: A study in patients and in vitro. Hepatol Res 38: 1112-1121, 2008.

36. Minemura M, Tanimura $\mathrm{H}$ and Tabor E: Overexpression of multidrug resistance genes MDR1 and cMOAT in human hepatocellular carcinoma and hepatoblastoma cell lines. Int J Oncol 15: 559-563, 1999.

37. Taniguchi K, Wada M, Kohno K, et al: A human canalicular multispecific organic anion transporter (cMOAT) gene is overexpressed in cisplatin-resistant human cancer cell lines with decreased drug accumulation. Cancer Res 56: 4124-4129, 1996. 